\title{
Photoluminescence detection of 2,4,6-trinitrotoluene (TNT) binding on diatom frustule biosilica functionalized with an anti-TNT monoclonal antibody fragment
}

Le Zhen $^{1}$, Nicole Ford ${ }^{2}$, Debra K. Gale ${ }^{1}$, Guritno Roesijadi ${ }^{2,3}$ and Gregory L. Rorrer ${ }^{1, *}$

${ }^{1}$ School of Chemical, Biological, and Environmental Engineering, Oregon State University, Corvallis, Oregon, 97331 USA

${ }^{2}$ Marine Sciences Laboratory, Pacific Northwest National Laboratory, Sequim, WA, 98382 USA

${ }^{3}$ Department of Microbiology, Oregon State University, Corvallis, Oregon, 97331 USA

*Corresponding author, rorrergl@engr.orst.edu

\begin{abstract}
A selective and label-free biosensor for detection of the explosive compound 2,4,6-trinitrotoluene (TNT) in aqueous solution was developed based on the principle of photoluminescence quenching of upon immunocomplex formation with antibody-functionalized diatom frustule biosilica. The diatom frustule is an intricately nanostructured, highly porous biogenic silica material derived from the shells of microscopic algae called diatoms. This material emits strong visible blue photoluminescence $(\mathrm{PL})$ upon UV excitation. PL-active frustule biosilica was isolated from cultured cells of the marine diatom Pinnularia sp. and functionalized with a single chain variable fragment (scFv) derived from an anti-TNT monoclonal antibody. When TNT was bound to the anti-TNT scFv-functionalized diatom frustule biosilica, the PL emission from the biosilica was partially quenched due to the electrophilic nature of the nitro $\left(-\mathrm{NO}_{2}\right)$ groups on the TNT molecule. The dose-response curve for immunocomplex formation of TNT on the scFv-functionalized diatom frustule biosilica had a half-saturation binding constant of $6.4 \pm 2.4 \cdot 10^{-8} \mathrm{M}$ and statistically-significant measured detection limit of $3.5 \cdot 10^{-8} \mathrm{M}$. The binding was selective for TNT and TNB (trinitrobenzene) but not RDX (hexahydro-1,3,5-trinitro-1,3,5-triazine) or 2,6-DNT (2,6-dinitrotoluene).
\end{abstract}

Keywords: antibody, biosensor, biosilica, diatom, photoluminescence, trinitrotoluene Revised manuscript BIOS-D-15-02291 submitted to Biosensors \& Bioelectronics 


\section{Introduction}

The manufacture and use of explosive compounds such as of 2,4,6-trinitrotoluene (TNT), combined with the general recalcitrance these compounds to chemical or biological remediation, has resulted in their widespread environmental contamination (Rodgers and Bunce, 2001). Furthermore, security concerns have heightened the need for detection of explosive compounds. Given the need for quantitative detection of TNT and related compounds in air, water, and soil, and on surfaces, new methods for detection of explosives are an active area of research (Caygill et al., 2012). Since most explosive compounds are not inherently fluorescent (Meaney et al., 2008), and typically are present at trace concentrations in complex matrices, indirect but selective detection methods are needed.

Biosensors are attractive for detection of TNT and other explosive compounds because the biological recognition element offers selectivity and sensitivity, particularly if antibodies designed to bind with TNT or related molecules are used (Smith et al., 2008). Towards this end, Goldman et al. have engineered single chain variable fragments (scFv) derived from monoclonal antibodies that bind TNT or other small molecule explosive compounds (Goldman et al., 2002, 2003, 2005; Medintz et al., 2004, Anderson et al., 2006). The anti-TNT scFv are easily expressed in Escherichia coli and can be modified by protein engineering to tailor their functionality and physicochemical properties. Recently, a scFv was genetically engineered for selectivity to TNT and trinitobenzene at $1 \mu \mathrm{g} / \mathrm{L}\left(4.4 \cdot 10^{-9} \mathrm{M}\right)$ sensitivity (Liu et al., 2013).

A major challenge for immunocomplex based biosensors is label-free detection (Smith et al., 2008; Holford et al., 2012), as enzyme-linked immunosorbent assay (ELISA) or other chemical labeling techniques are typically required for optical detection. Current label-free platforms for immuno-complex based sensing rely on surface plasmon resonance (SPR) (Fan et al., 2008), piezoelectric (Smith et al., 2008), electrochemical, or impedance (Daniels and Pourmand, 2007) measurements, all of which require specialized instrument packages.

In previous work, we discovered that selective and label-free optical detection of antibodyantigen complex formation could be measured through visible blue photoluminescence (PL) 
intensity change of antibody-functionalized diatom frustule biosilica under UV light (Gale et al., 2009). The diatom frustule is an intricately nanostructured, highly porous biogenic silica material that constitutes the shell of microscopic algae called diatoms. This material emits a strong, visible blue photoluminescence upon UV excitation, and possesses surface silanol groups which can be readily functionalized (Qin et al., 2008; Gale et al., 2009).

In this study, we functionalized PL-active frustule biosilica isolated from cultured diatom cells with an anti-TNT scFv of sequence described by Liu et al (2013) to make a TNT biosensor based on photoluminescence detection. Since photoluminescence quenching is observed when electrophilic TNT molecules interact with PL-active materials (Content et al., 2000), the selective detection of TNT binding with scFv-functionalized diatom frustule biosilica was measured by this principle. We report the fabrication, selectivity, and sensitivity of this new, label-free TNT biosensor below.

\section{Methods}

\subsection{Diatom cell culture}

The marine diatom Pinnularia sp. was obtained from the UTEX Culture Collection of Algae (UTEX \#B679) and cultivated in $100 \mathrm{~mL}$ of autoclaved Harrison's artificial seawater medium (Harrison et al., 1980) enriched with $0.5 \mathrm{mM} \mathrm{Na}_{2} \mathrm{SiO}_{3}$ in $500 \mathrm{~mL}$ foam-stoppered glass flasks. Cultures were incubated in $22^{\circ} \mathrm{C}$ under $150 \mu \mathrm{E} \mathrm{m} \mathrm{m}^{-2}$ incident light intensity on a $14 \mathrm{~h} / 10 \mathrm{~h}$ light/dark cycle for 21 days. To facilitate gas exchange, each flask was swirled for for 10 s once per day.

\subsection{Biosilica frustule isolation}

Only diatom cells which were cultured for at least 14 days to stationary were used for biosilica isolation to ensure that the biosilica was PL-active (Qin et al. 2008). Biosilica frustules were isolated from cultured diatom cells by hydrogen peroxide treatment reported previously (Jeffyres et al., 2008; Gale et al., 2009), with the following modifications as described in the Supplementary material. 


\subsection{Production of scFv}

The single chain variable fragment (scFv) derived from the monoclonal antibody 2G5B5 (Liu et al, 2013) was expressed in E. coli and purified as described in the Supplementary material. This anti-TNT ScFv has a molecular weight of $28.2 \mathrm{kDa}$ with 252 amino acid residues, 42 of which contain free amine groups.

\subsection{Functionalization of diatom biosilica}

The anti-TNT scFv was covalently bonded to the diatom biosilica frustules by a three-step process which involved amination of surface silanol groups on the diatom biosilica, attachment of the amine crosslinking reagent disuccinimydylsuberate (DSS) to the amine-functionalized diatom biosilica, and covalent attachment of DSS to the scFv at one of its free lysine groups (Fig.

1). Aminated diatom biosilica was prepared by reaction with 3-aminopropyltrimethoxysilane (APTMS) as described previously (Gale et al., 2009) with the following modifications. The aminated biosilica was washed twice with deionized water and re-suspended in deionized water to a very dilute suspension of $1.0 \mathrm{mg}$ biosilica/mL, which was easy to transfer by pipette. A $10 \mu \mathrm{L}$ aliquot of the biosilica suspension was pipetted on the center of a $22 \mathrm{~mm} \times 22 \mathrm{~mm}$ acidwashed cover glass slip. The droplet suspension spread to diameter of $5 \mathrm{~mm}$, and the water was allowed evaporate at room temperature under flowing sterile air within a laminar flow hood to prevent particle contamination. This deposition process was repeated three additional times to form a particulate film containing $40 \mu \mathrm{g}$ biosilica. The film was dried in air at $90{ }^{\circ} \mathrm{C}$ for $1 \mathrm{~h}$ and stored under dessication at room temperature. Diatom biosilica is known to possess silanol groups (Qin et al., 2008) which carry a negative charge and promote the adhesion of the frustules to the surface and to each other.

Disuccinimidyl suberate (DSS, Pierce Biotechnology, $368.35 \mathrm{~g} / \mathrm{mol}$ ) served as the crosslinking agent for covalently attaching the scFv onto the aminated diatom biosilica. Furthermore, there was also the possibility that the crosslinking reaction also connected diatoms to one another (Fig. 1 inset). DSS was dissolved in anhydrous acetonitrile (ACN) to concentration of $1.0 \mathrm{mg} / \mathrm{L}$ under $0.4 \mathrm{~nm}$ molecular sieve pellets for 2 days to remove water. A $10 \mu \mathrm{L}$ aliquot of the DSS solution was pipetted on each aminated biosilica film and allowed to react for $10 \mathrm{~min}$. The DSSfunctionalized biosilica film was then dip-rinsed in phosphate-buffered saline (PBS, pH 7.4), and 
the entrained PBS was removed by wicking the film with a paper towel. Then, $10 \mu \mathrm{L}$ of anti-TNT scFv antibody solution $(0.6 \mathrm{mg} / \mathrm{mL})$ was immediately pipetted on each DSS functionalized biosilica film. The films were then transferred into sealed Petri dishes (60 x $15 \mathrm{~mm})$ humidified with a water-saturated paper towel, and the scFv crosslinking reaction was carried out on an orbital shaker at $100 \mathrm{rpm}$ for $2 \mathrm{~h}$. After the reaction, the scFv-functionalized biosilica films were dip-rinsed in PBS for three times to remove excess ScFv, and the entrained PBS was removed. The scFv-functionalized biosilica thin film deposits were dried in stagnant air and room temperature for exactly $1 \mathrm{~h}$ before photoluminescence $(\mathrm{PL})$ measurement. In one set of samples using this procedure, the scFv concentration in the rinse solution was assayed spectrophotometrically by the Bradford method to estimate the scFv loading on the diatom biosilica. In order to provide a control substrate, the protein Bovine Serum Albumin (BSA) was covalently bonded to Pinnularia diatom biosilica by the same methods described above.

\subsection{Photoluminescence spectroscopy}

PL emission measurements on the diatom thin film deposits under UV laser excitation were adapted from our previous work (Gale et al., 2009), with modifications as described in the Supplementary material.

\subsection{Challenge of scFv-functionalized diatom biosilica with TNT}

The scFv-functionalized biosilica films were challenged with TNT solution immediately after PL measurement. To prepare the TNT solution, 2,4,6-trinitrotoluene (Chem Service Inc., Lot\# 439200, min $30 \mathrm{wt} \%$ water, purity $99.1 \%, 227.13 \mathrm{~g} / \mathrm{mol}$ ) was first dissolved in anhydrous acetonitrile (ACN) to make $1.00 \mathrm{mg} T N T / \mathrm{mL}$ stock solution. The stock solution was diluted with PBS buffer to a series of TNT test solutions ranging from $4.8 \cdot 10^{-9} \mathrm{M}(1.09 \mu \mathrm{g} / \mathrm{L})$ to $4.8 \cdot 10^{-7} \mathrm{M}$ TNT $(109 \mu \mathrm{g} / \mathrm{L})$. In parallel, a blank solution containing $1.0 \mathrm{mg} / \mathrm{mL}$ ACN diluted in PBS buffer at each dilution ratio served as the zero reference. A $15 \mathrm{~mL}$ aliquot of a given TNT test solution or complimentary blank solution was added to a single $60 \times 15 \mathrm{~mm}$ Petri dish. Then, one scFvfunctionalized biosilica film was placed in the Petri dish and contacted with the TNT test solution for $2 \mathrm{~h}$ under $50 \mathrm{rpm}$ orbital shaking at room temperature. After contact, the films 
were dip-rinsed with PBS buffer three times. Excess PBS was removed with a paper towel, and the films were dried in stagnant air and room temperature for exactly $1 \mathrm{~h}$ before PL measurement. All TNT challenge experiments were performed in triplicate at given TNT concentration, and consisted of two sets of PL spectra, one before TNT challenge, and one after. The ratio of TNT molecules to binding sites was such that TNT consumption from the liquid phase could be neglected.

The PL response to the TNT challenge of the scFv-functionalized diatom biosilica film was quantified integrating the spectra, normalizing with respect to the scFv-functionalized biosilica, and subtracting the zero reference. The normalized PL response $(\mathrm{Q})$ is defined by the following relationships:

$$
\begin{gathered}
Q=\frac{\int_{\lambda_{1}}^{\lambda_{2}} I_{s c F V}(\lambda) d \lambda-\int_{\lambda_{1}}^{\lambda_{2}} I_{s c F V-T N T}(\lambda) d \lambda}{\int_{\lambda_{1}}^{\lambda_{2}} I_{s c F V}(\lambda) d \lambda}-Q_{o} \\
Q_{o}=\frac{\int_{\lambda_{1}}^{\lambda_{2}} I_{s c F V}(\lambda) d \lambda-\int_{\lambda_{1}}^{\lambda_{2}} I_{s c F V-A C N, o}(\lambda) d \lambda}{\int_{\lambda_{1}}^{\lambda_{2}} I_{s c F V}(\lambda) d \lambda}
\end{gathered}
$$

where $I(\lambda)$ is the PL emission intensity vs. wavelength with subscripts scFv (scFv-functionalized diatom biosilica), scFv-TNT (scFv-diatom biosilica after TNT challenge and PBS rinsing), scFv-ACN (scFv-biosilica after challenge with zero TNT reference), and $\lambda_{1}$ and $\lambda_{2}$ are the wavelengths over which the PL spectra were integrated (400 and $450 \mathrm{~nm}$ respectively). The integration smoothed the data, and the normalization provided a positive value for $Q$ under conditions where the PL response for scFv-functionalized biosilica was greater than that for TNT immunocomplex on scFv-functionalized diatom biosilica. The blank for each set of measurements $\left(Q_{0}\right)$ was required because the PL response of ScFv-functionalized diatom biosilica increased after soaking in PBS buffer.

To assess for the specificity of TNT detection by PL measurement of the immuncomplex formation, the scFv-functionalized diatom biosilica was challenged with series of munitions constituents. These included 1,3,5-trinitrobenzene (TNB, SUBELCO, Lot\#LB91318, $1000 \mu \mathrm{g} / \mathrm{mL}$ in ACN, 213.10g/mol), hexahydro-1,3,5-trinitro-1,3,5-triazine (RDX, Cerilliant Corporation, Lot\# 
ER12012-01, $1000 \mu \mathrm{g} / \mathrm{mL}$ in ACN, $222.12 \mathrm{~g} / \mathrm{mol}$ ), 2,6-dinitrotoluene (2,6-DNT, Cerilliant Corporation, Lot\# FN092811-01, 1000 mg/mL in ACN, $182.13 \mathrm{~g} / \mathrm{mol}$ ), which were individually added to PBS buffer solution to concentration of $4.8 \cdot 10^{-7} \mathrm{M}$, and tested as described above. Each solution was spiked with $4.8 \cdot 10^{-7} \mathrm{M}$ TNT and tested in parallel. Finally, TNT at high concentration of $4.4 \cdot 10^{-4} \mathrm{M}$ was challenged with non-functionalized diatom biosilica as a substrate control, as detailed in the Supplementary material.

\subsection{Scanning electron microscopy (SEM)}

Pinnularia diatom biosilica films prepared above were sputter-coated with 60/40 gold/platinum for $30 \mathrm{~s}$, and imaged using an FEI Quanta 600 FEG scanning electron microscope at $10 \mathrm{kV}$.

\subsection{Epi-fluorescence microscopy}

Alex Fluor 555 labeled trinitrobenze (AF-TNB) served as a fluorescently labeled TNT surrogate with synthesis as described in the Supplementary material. The scFv-functionalized Pinnularia diatom biosilica thin films prepared above were soaked in Tris buffer ( $\mathrm{pH} 7.4$ ) for 3 days, then challenged with $2 \mathrm{~mL}$ of $25 \mathrm{nM}$ AF-TNB dissolved in PBS buffer using the methods described above for the TNT challenge experiments. After rinsing for 3 times in PBS buffer and drying in air for one day, white light images and their corresponding epi-fluorescence images of the AFTNB challenged biosilica films were taken with a Leica DM inverted light microscope equipped with CY3 filter (excitation $530 / 50 \mathrm{~nm}$, emission $610 / 75 \mathrm{~nm}$ ) at $40 \mathrm{X}$ magnification with 2 s integration time.

\section{Results}

\subsection{SEM Imaging of diatom biosilica}

SEM images of the Pinnularia diatom frustule thin film and fine structure of the diatom biosilica are presented in Figs. 2a-c. The frustules were largely intact and randomly distributed on the surface, with both value and girdle band structures evident. The hydrogen peroxide treatment of the diatom cells generally split apart the upper and lower theca of the frustule. Pinnularia is a pennate diatom with ellipsoidal shape with major and minor axes of approximately $20 \mu \mathrm{m}$ and $6 \mu \mathrm{m}$ respectively. The surface consists of pores in rectangular array approximately $200 \mathrm{~nm}$ 
in diameter spaced 300-400 $\mathrm{nm}$ apart. At the base of each pore were 4-5 nanopores approximately $50 \mathrm{~nm}$ in diameter.

\subsection{Epifluorescence imaging of scFv-functionalized diatom biosilica}

Fig. $2 d$ presents an epifluorescence image of the anti-TNT scFv-functionalized Pinnularia diatom thin film after challenge with Alexa Fluor 555 labeled TNB followed by rinsing in PBS buffer. Pinnularia diatom thin films that were not functionalized with scFv showed no epifluorescence after challenge with Fluor 555 labeled TNB followed by rinsing in PBS buffer. The loading of the anti-TNT scFv loaded on the diatom biosilica after functionalization was $0.041 \pm 0.002 \mathrm{mg}$ $\mathrm{scFv} / \mathrm{mg} \mathrm{SiO} 2$ (propagated error $1.0 \mathrm{SD}, \mathrm{n}=3$ ) as determined by spectrophotometric assay before and after functionalization. If it assumed that the anti-TNT scFv is approximately $4 \mathrm{~nm}$ in diameter (Goldman et al., 2005), and the internal surface area of Pinnularia diatom biosilica isolated by hydrogen peroxide treatment is approximately $30 \mathrm{~m}^{2} / \mathrm{g}$ (Van Eynde et al., 2014), then the estimated scFv coverage is approximately $2.5 \cdot 10^{4}$ molecules per $\mu \mathrm{m}^{2}$, or $40 \%$ of the total microporous surface area.

\subsection{PL spectra from scFv-functionalized diatom biosilica}

Representative photoluminescence (PL) emission spectra from the aminated Pinnularia diatom frustule biosilica and anti-TNT scFv-functionalized diatom biosilica using the DSS crosslinker are compared in Fig. 3. The PL emission under $337 \mathrm{~nm}$ UV laser excitation was centered in the visible blue range. The peak PL response was normalized to 1.0 for the scFV-functionalized diatom biosilica. The addition of the scFv increased the peak PL emission intensity of the amine-functionalized diatom biosilica by about a factor of 3 , due to the presence of nucleophilic amine $\left(-\mathrm{NH}_{2}\right)$ groups within the antibody, as observed in our previous work (Gale et al., 2009). The PL spectra of the immunocomplex between TNT and ScFv-functionalized diatom biosilica are also compared in Fig. 3. The TNT immunocomplex decreased the PL emission intensity of scFv-functionalized diatom biosilica due to the presence of electrophilic $\mathrm{NO}_{2}$ groups on TNT. After the TNT challenge, all samples were washed three times in PBS buffer to remove nonspecific adsorption. The peak wavelength for the APTMS-functionalized diatom biosilica was 
near $420 \mathrm{~nm}$ vs. $415-420 \mathrm{~nm}$ for scFv-functionalized diatom biosilica. TNT immunocomplex formation did not change the peak PL wavelength, only the intensity of the PL emission.

To verify that partial quenching of the PL response was due to the interaction of TNT with the diatom biosilica substrate itself, the PL response after free adsorption of TNT on nonfunctionalized diatom biosilica without washing was also measured, as shown in the Supplementary material (Fig. S1).

\subsection{Dose-response curve for PL-based detection of TNT immunocomplex formation}

The decrease in PL emission intensity served as the basis for label-free TNT detection. The normalized, integral PL response (Q), defined by equation (1), was used to quantify this relative PL change at a given TNT concentration. The dose-response curve of the PL response vs. initial TNT concentration from $4.8 \cdot 10^{-9}$ to $4.8 \cdot 10^{-7} \mathrm{M}(\mathrm{mol} / \mathrm{L})$ TNT is presented in Fig. 4 . The data were fitted to the saturation model of the form

$$
Q=\frac{C_{T N T}^{n} Q_{\max }}{K_{T N T}^{\prime}+C_{T N T}^{n}}
$$

where $Q_{\max }$ is the normalized PL response at saturation, $K_{T N T}^{\prime}$ is the binding constant, $C_{T N T}$ is the TNT concentration (M), and $n$ is order on $\mathrm{C}_{\mathrm{TNT}}$. If $n=1$, then the model reduces to the Langmuir adsorption isotherm with binding constant $K_{T N T}$ in consistent units with $C_{T N T}$. If $n=2$, then the

binding constant is determined by $K_{T N T}=\sqrt{K_{T N T}^{\prime}}$. The fitted parameters, using the Marquardt method of least squares nonlinear parameter estimation, are presented in Table 1 . The model with $n=2$ fitted the data at low concentrations better, but the model at $n=1$ reduced the error for estimation of $K_{T N T}$.

\subsection{Specificity of immunocomplex detection on scFv-functionalized diatom biosilca}

The potential for immunocomplex formation of scFv-functionalized diatom frustule biosilica with other munitions constituents, including RDX, TNB, and 2,6-DNT, is presented in Fig. 5. In all experiments, each concentration was fixed at $4.8 \cdot 10^{-7} \mathrm{M}$, which was near the saturation concentration for TNT. After immuncomplex formation, each film was soaked and rinsed in PBS buffer for 3 times to mitigate the possibility of nonspecific TNT absorption. Each sample was 
also spiked with $4.8 \cdot 10^{-7} \mathrm{M}$ TNT. The results were compared statistically by ANOVA, as shown in Table 2. The analysis shows that RDX is not detected by measurement of PL response, and that TNT and TNB response were statistically indistinguishable. Detection of 2,6-DNT was possible, but the response was not as strong as for TNT or TNB. Finally, the presence of 2,4DNT or RDX did not affect the TNT response at the saturation condition. Methods to wash off the bound compounds and reuse the sensor platform were not considered by this study.

To determine the potential for nonspecific adsorption on diatom biosilica and amino acid residues, the Pinnularia diatom biosilica was functionalized with the protein BSA and challenged with 4.8.10 $10^{-7} \mathrm{M}$ TNT as described above, and then rinsed one time in PBS buffer. The normalized PL response was $Q=0.015 \pm 0.018$ (1.0 S.E.), which although greater than zero was not statistically distinguishable $(p=0.371)$ from the blank control.

\section{Discussion}

The presence of silanol groups on the diatom biosilica surface on frustules isolated from cultured diatom cells (Qin et al., 2008) enables its chemical functionalization (DeStefano et al., 2009; Gale et al., 2009), including covalent bonding of antibodies to the frustule surface. In this present work, we functionalized Pinnularia frustule biosilica isolated from cultured diatom cells with a single chain variable fragment (scFv) from anti-TNT monoclonal antibody (Figs. 1, 2d).

Photoluminescence results in the spontaneous emission of light from a solid material surface under excitation by light of a higher energy within the conduction band of the material (Gfroerer, 2000). PL emission in the visible blue range by diatom biosilica under UV irradiation is attributed to the nanostructured, highly-porous surface morphology of the frustule that reveals surface silicon oxide defects characteristic of biogenic $\mathrm{SiO}_{2}$, including silanol groups and non-bridging oxygen centers (DeStefano et al., 2005; Qin et al., 2008). PL emission from a material surface is affected by its interaction with other molecules, where nucleophilic molecules enhance the PL emission (Gale et al., 2009) and electrophilic molecules quench the PL emission (Setaro et al., 2007) on the diatom frustule biosilica. 
The concept of PL detection of the immunocomplex is schematically shown in Fig. 6 . The likely origin of decreased PL emission of the scFv-functionalized diatom biosilica in response to immuncomplex formation with TNT was the presence of three electrophilic nitro $\left(-\mathrm{NO}_{2}\right)$ groups per molecule of TNT. Based on the known amino acid sequence of the anti-TNT scFv, there are 42 nucleophilic $-\mathrm{NH}_{2}$ groups per scFv molecule. Consequently, only partial quenching of $\mathrm{PL}$ was possible based on binding of electrophilic TNT with the nucleophilic anti-TNT scFv, and the extent of quenching relative to the scFv-functionalized biosilica is most likely related to the $\mathrm{NO}_{2} / \mathrm{NH}_{2}$ group ratio. At saturation, the relative decrease in the integrated PL response was around $13-15 \%$ (Fig. 3, Table 1), whereas the $\mathrm{NO}_{2} /-\mathrm{NH}_{2}$ group ratio of the immunocomplex was 0.071. This ratio may set the extrinsic limit to the signal sensitivity. In this context, the use of smaller TNT bioreceptors like dodecapeptides (Goldman et al., 2002) would decrease the number of nucleophilic groups, increase the $\mathrm{NO}_{2} / \mathrm{NH}_{2}$ group ratio, and hence could potentially increase the change in signal intensity. Based on the discussion above, in principle the PL signal would significantly increase if the TNT-bound scFv on the diatom biosilica was challenged again with its complimentary scFv in a competitive assay format. However, in this instance, this scFv would be essentially acting as a label. Since the objective of this work was to explore a label-free detection platform, this approach was not considered.

In previous work, the nonspecific chemisorption of nitrogen dioxide $\left(\mathrm{NO}_{2}\right)$ gas on the surface of diatom biosilica partially quenched the PL emission (Setaro et al., 2007). Based on timeresolved photoluminescence measurements for the lifetime of the excited states, it was proposed that surface oxygen vacancies states acted as excited states for radiative transitions, and their interaction with electrophilic $\mathrm{NO}_{2}$ molecules may have suppressed the density of radiative states (Lettieri et al., 2008). The partial quenching of PL upon immuncomplex formation of TNT with scFv-functionalized diatom biosilica may be expected to proceed by same mechanism.

The dose-response curve for TNT immunocomplex formation with scFv-functionalized diatom biosilica frustule had a half-saturation binding constant of $6.4 \pm 2.4 \cdot 10^{-8} \mathrm{M}$ (1.0 S.E., Table 1), compared with $1.6 \cdot 10^{-8} \mathrm{M}$ for binding of TNT with the anti-TNT scFv in solution (Liu et al., 
2013). The measured and statistically significant limit of quantitative, label-free detection was approximately $3.5 \cdot 10^{-8} \mathrm{M}$, although average nonzero detection signals were as low as $4.8 \cdot 10^{-9}$ $\mathrm{M}$, nominally $10 \%$ of the binding constant (Fig. 4). In comparison, the detection limit of the anti-TNT scFv in solution for a label-based competitive immunoassay was $4.4 \cdot 10^{-9} \mathrm{M}$ (Liu et al., 2013).

The anti-TNT scFv-functionalized diatom frustule biosensor for detection of munitions constituents based in immunocomplex formation was selective for TNT and TNB but not RDX or 2,6-DNT. This result is consistent with the molecular design of the anti -TNT ScFv, where recombinant antibody fragments derived from phage display were selected to create $\pi-\pi$ interaction between the docking site and trinitroaromatic structures (Goldman et al., 2003, 2005), not to cyclic nitramine structures or dinitroaromatic structures. Based on this result, the biosensing layer is limited to detection of trinitroaromatic structures due to the nature of the immunocomplex with the scFv. It was beyond the scope of this study to more broadly investigate the PL quenching associated with immunocomplex formation by a series of antibodies with their complimentary electrophilic antigens.

TNT is known to interact with APTMS-functionalized silica (Gao et al., 2008), and control experiments showed that nonspecific adsorption of TNT on diatom biosilica decreased the PL response. However, in the immunocomplex experiments with scFv-functionalized diatom biosilica, although nonspecific TNT adsorption might have occurred, it did not survive the $3 x$ PBS buffer rinsing process.

TNT is known to quench PL emission following adsorption on optically active materials such as porous silicon (Content et al., 2000) or organosilicon (Martinez et al., 2012). Previous work with photoluminescence-based TNT detection in aqueous environments was either not selective or required chemical labeling. Sohn et al. (2001) used organosilicon polymers (e.g. polytetraphenylsilole) to detect TNT in seawater based on partial quenching of fluorescence by absorbed TNT with detection limit of $2.2 \cdot 10^{-7}$ M TNT, and Feng et al. (2009) used conjugated polymer-grafted silica nanoparticles to detect TNT in solution by partial quenching of 
fluorescence with detection limit of $1.0 \cdot 10^{-6} \mathrm{M}$ TNT. For selective TNT recognition, Goldman et al. (2005) tethered anti-TNT to CdSe-ZnS core-shell quantum dots (QDs), where a dyelabeled TNB pre-bound in the antibody binding site quenched the QD photoluminescence via proximity-induced fluorescence resonance energy transfer (FRET), which was restored after displacement via TNT challenge (Goldman et al. 2005), and provided a detection limit of $8.8 \cdot 10^{-}$

${ }^{8} \mathrm{M}$. In this study, the TNT detection limit was comparable to or better than all of the studies cited above.

\section{Conclusions}

This study describes the fabrication and performance of an antibody-based biosensor which provides sensitive, selective, and label-free detection of TNT based on the measurement principle of partial quenching of photoluminescence emission arising from immunocomplex formation of electrophilic $-\mathrm{NO}_{2}$ moieties with the anti-TNT scFv-functionalized diatom biosilica. This biosensor offers label-free detection on a PL-active, transparent, and environmentallybenign solid substrate. In this regard, the anti-TNT ScFv monoclonal antibody fragment is covalently bound to a biologically derived, nanostructured biosilica, which can be produced by diatom cell culture to ensure controlled properties. Furthermore, the immunocomplex does not require any additional chemical labeling to enable TNT detection, since the origin of the PL signal is from the nanostructured diatom biosilica itself.

\section{Acknowledgements}

Support of this research was provided by U.S. Department of Defense, Office of Naval Research, Synthetic Biology Program, Award Numbers N0001414IP20004 (PNNL) and N000141210313 (OSU). Drs. Ellen Goldman and Igor Medintz, U.S. Naval Research Laboratory (NRL), Washington DC, kindly provided the plasmid containing the unmodified anti-TNT scFv, and the AF555-TNB standard. 


\section{References}

Anderson, G.P., Moreira, S.C., Charles, P.T., Medintz, I.L., Goldman, E.R., Zeinali, M., Taitt, C.R., 2006. Anal. Chem. 78, 2279-2285.

Caygill, S.J., Davis, F., Higson, S.P.J., 2012. Talanta 88, 14-29.

Content, S., Trogler, W.C., Sailor, M.J., 2000. Chem. Eur. J. 6, 2205-2213.

Daniels, D.S., Pourmand, N., 2007. Electroanal. 19, 1239-1257.

De Stefano, L., Rotiroti, L., De Stefano, M., Lamberti, A., Lettieri, S., Setaro, A., Maddalenad, P., 2009. Biosens. Bioelectron. 24, 1580-1584.

DeStefano, L., Rendina, I., De Stefano, M., Bismuto, A., Maddalena, P., 2005. Appl. Phys. Lett. 87, 233902.

Fan, X., White, I.M., Shopova, S.I., Zhu, H., Suter, J.D., Sun, Y., 2008. Anal. Chim. Acta 620, 8-26. Feng, J., Li, Y., Yang, M. , 2010. Sensor Actuat B-Chem 145, 438-443.

Gale, D.K, Gutu, T., Jiao, J., Chang, C.-H., Rorrer, G.L., 2009. Adv. Funct. Mater. 19, 926-933.

Gao, D., Wang, Z., Liu, B., Ni, L., Wu, M., Zhang, Z., $2008 . \quad$ Anal. Chem. 80, 8545-8553.

Gfroerer, T.H., 2000. Photoluminescence in analysis of surfaces and interfaces, in: Meyers, R.A. (Ed.), Encyclopedia of Analytical Chemistry, John Wiley \& Sons Ltd, Chichester, pp. 92099231.

Goldman, E.R., Hayhurst, A., Lingerfelt, B.M., Iverson, B.L., Geogiou, G., Anderson, G.P., 2003. J. Environ. Monit. 5, 380-383.

Goldman, E.R., Medintz, I.L., Whitley, J.L., Hayhurst, A., Clapp, A.R., Uyeda, H.T., Deschamps, J.R., Lassman, M.E., Mattoussi, H., 2005. J. Am. Chem. Soc. 127, 6744-6751.

Goldman, E.R., Pazirandeh, M.P., Charles, P.T., Balighian, E.D., Anderson, G.P., 2002. Anal. Chim. Acta 457, 13-19.

Harrison, P.J., Waters, R.E., Taylor, F.J.R., 1980. J. Phycol. 16, 28-35.

Holford, T.R.J., Davis, F., Higson, S. P.J., 2012. Biosens. Bioelectron. 34, 12-24

Jeffryes, C., Gutu, T., Jiao, J., and Rorrer, G.L., 2008. Mat. Sci. Eng. C 28, 107-118.

Lettieri, S., Setaro, A., De Stefano, L., De Stefano, M., Maddalena, P., 2008. Adv. Funct. Mater. $18,1257-1264$.

Liu, J.L., Zabetakis, D., Acevedo-Velez, G., Goldman, E.R., 2013. Anal. Chim. Acta 759, 100-104. Martinez, H.P., Grant, C.D., Reynolds, J.G., Trogler, W.C., 2012. J. Mater. Chem. 22, 2908-2914. 
Meaney, M.S., McGuffin, V.L., 2008. Anal Bioanal Chem 391, 2557-2576.

Medintz, I.L., Goldman, E.R., Lassman, M.E., Hayhurst, A., Kusterbeck, A.W., Deschamps, J.R., 2004. Anal. Chem. 77, 365-372.

Medintz, I.L., Goldman, E.R., Lassman, M.E.,Mauro, J.M., 2003. Bioconjugate Chem. 14, 909918.

Qin, T., Gutu, T., Jiao, J., Chang, C.-H., Rorrer, G.L., 2008. J. Nanosci. Nanotechnol. 8, 2392-2398.

Rodgers, J.D., Bunce, N.J., 2001. Wat. Res. 35, 2101-2111

Setaro, A., Lettieri, S., Maddalena, P., DeStefano, L., 2007. Appl. Phys. Lett. 91, 051921.

Smith, R.G., D'Souza, Nicklin, S., 2008. Analyst 133, 571-584.

Sohn, H., Calhoun, R.M., Sailor, M.J., Trogler, W.C., 2001. Angew. Chem. Int. Ed. 40, 2104-2105.

Van Eynde, E., Lenaerts, B., Tytgat, T., Verbruggen, S.W., Hauchecorne, B., Blustc, R., Lenaertsa, S., 2014. RSC Adv. 4, 56200-56206. 


\section{Figure Legends}

Fig. 1. Schematic of biosilica functionalization by scFv.

Fig. 2. Imaging of Pinnularia frustules. (a-c) SEM images showing diatom frustule thin film layer, single diatom frustule, and frustule pore array; (d) epi-fluorescence imaging of scFvfunctionalized diatom biosilica after immunocomplex formation with Alexa Fluor 555 labeled TNB.

Fig. 3. Comparison of PL emission spectrum from APTMS-functionalized Pinnularia diatom biosilica frustules (BS), scFv antibody functionalized diatom biosilica crosslinked with disuccinimydylsuberate (BS-scFv), and scFv-functionalized diatom biosilica after immunocomplex formation with $4.8 \cdot 10^{-7} \mathrm{M}$ 2,4,6-trinitrotoluene (BS-scFv+TNT). All spectra were normalized relative to scFv-functionalized diatom biosilica.

Fig. 4. Dose-response curve for challenge of TNT with scFv-functionalized Pinnularia diatom frustule thin films after 3 rinsing steps. The dotted line refers to the fit of the data with Langmuir model with $n=1$, and the solid line refers to the fit of the data to the Langmuir model with $n=2$. All error bars represent propagated errors with $+/-1.0$ standard error. Fitted parameters are provided in Table 1.

Fig. 5. Comparison of munition constituent detection of scFv-functionalized Pinnularia diatom frustule thin films after 3 rinsing steps. All error bars represent propagated errors with +/- 1.0 standard deviation (1.0 SD, $n=3$ ). Inset images provide molecular structure of each munition constituent. Statistical analysis comparison by ANOVA is provided in Table 2.

Fig. 6. Conceptual diagram of photoluminescence quenching upon binding of TNT with the scFv-functionalized diatom frustule. 
Table 1

Fitted parameters from dose response curve for TNT challenge with scFv-functionalized Pinnularia diatom frustule thin films (data provided in Fig. 4).

\begin{tabular}{ccccc}
\hline & $\begin{array}{c}\text { Model 1 } \\
(\mathrm{n}=1)\end{array}$ & & \multicolumn{2}{c}{$\begin{array}{c}\text { Model 2 } \\
(\mathrm{n}=2)\end{array}$} \\
\cline { 2 - 5 } Parameter & Value & 1.0 S.E. & Value & 1.0 S.E. \\
\hline $\mathrm{Q}_{\max }$ & 0.146 & 0.022 & 0.130 & 0.013 \\
$\mathrm{~K}_{\mathrm{TNT}}$ & $6.4 \cdot 10^{-8}$ & $2.4 \cdot 10^{-8}$ & $1.8 \cdot 10^{-15}$ & $2.2 \cdot 10^{-15}$ \\
$\mathrm{r}^{2}$ & 0.84 & & & 0.90 \\
$\left(\mathrm{~K}_{\mathrm{TNT}}\right)^{1 / \mathrm{n}}(\mathrm{M})$ & $6.4 \cdot 10^{-8}$ & $2.4 \cdot 10^{-8}$ & $4.2 \cdot 10^{-8}$ & $4.7 \cdot 10^{-8}$ \\
\hline
\end{tabular}

\section{Table 2}

ANOVA analysis for binding of various munitions constituents to scFv-functionalized Pinnularia diatom biosilica (data provided in Fig. 5).

\begin{tabular}{ccccccc}
\hline \multicolumn{7}{c}{ ANOVA Single Factor $(p$-value at $\alpha=0.05)$} \\
\cline { 2 - 7 } Challenge & TNB & RDX & 2,6 -DNT & TNB+TNT & RDX+TNT & $2,6-$ DNT+TNT \\
\hline TNT vs. & 0.37 & $6.6 \cdot 10^{-4}$ & $2.1 \cdot 10^{-4}$ & 0.035 & 0.19 & 0.82 \\
TNB vs. & - & $7.3 \cdot 10^{-3}$ & $4.3 \cdot 10^{-3}$ & - & 0.15 & 0.064 \\
RDX vs. & - & - & 0.033 & $1.2 \cdot 10^{-3}$ & - & $4.1 \cdot 10^{-3}$ \\
\hline
\end{tabular}


Fig. 1

\section{diatom biosilica}

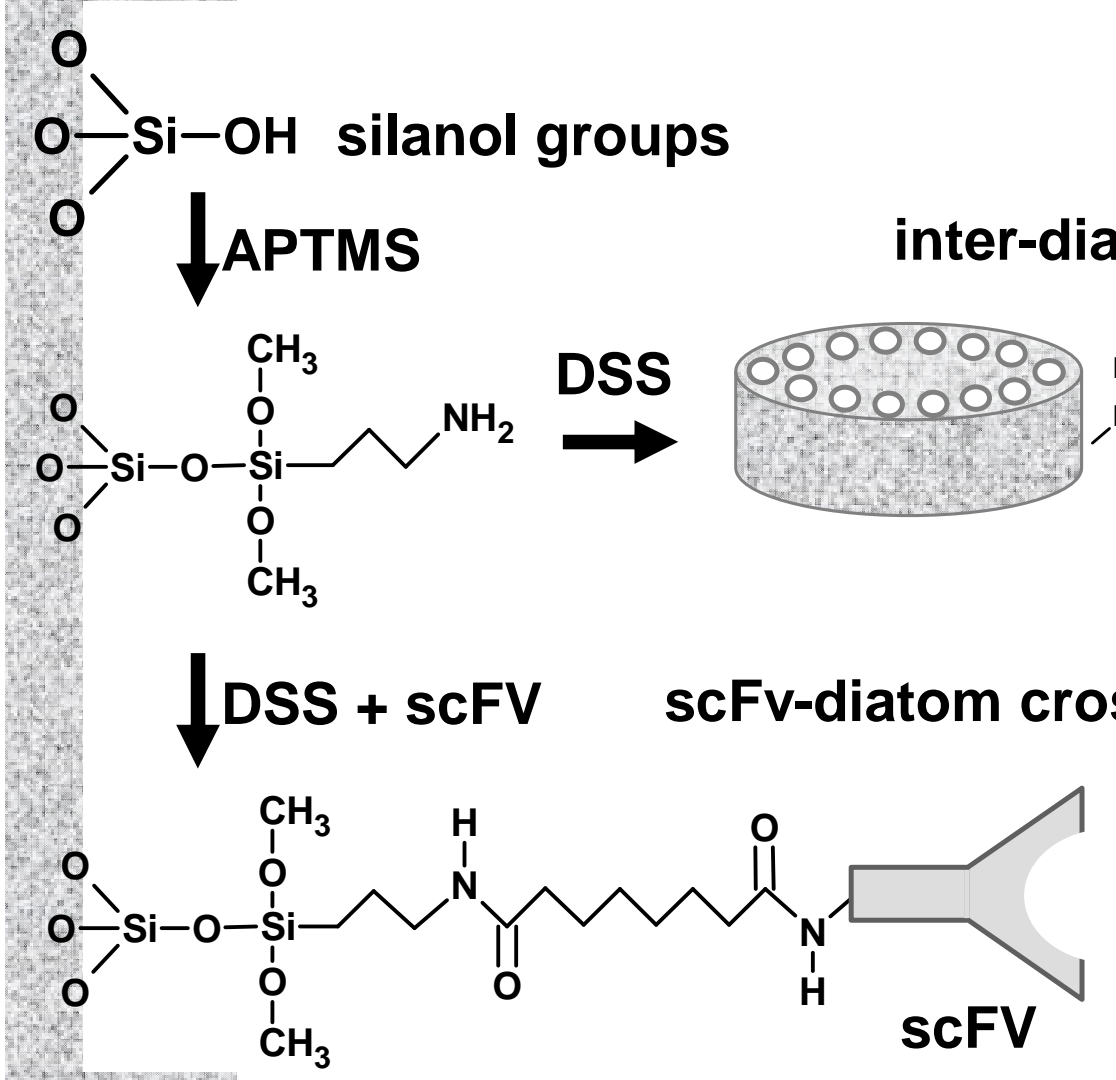


Fig. 2
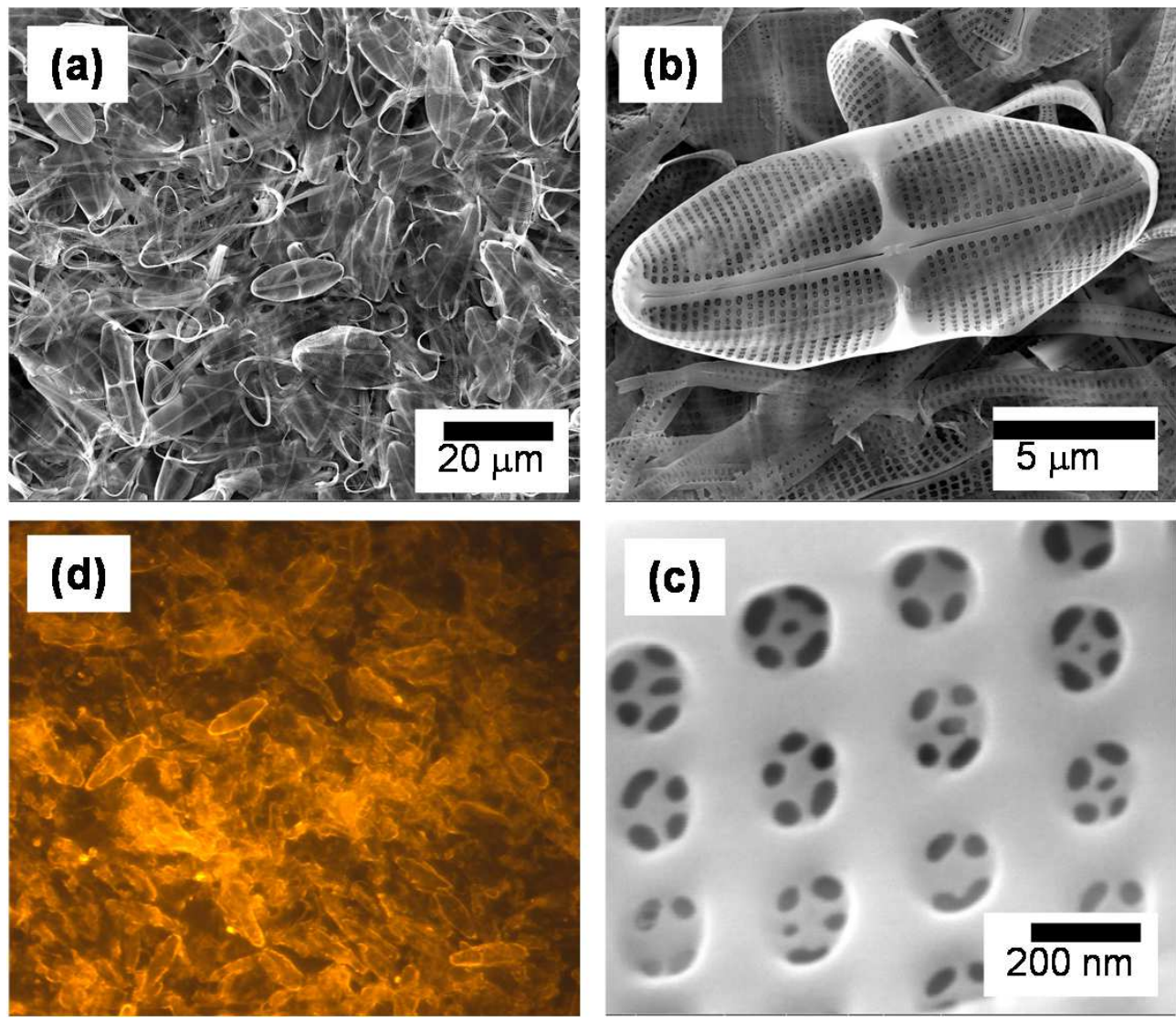
Fig. 3

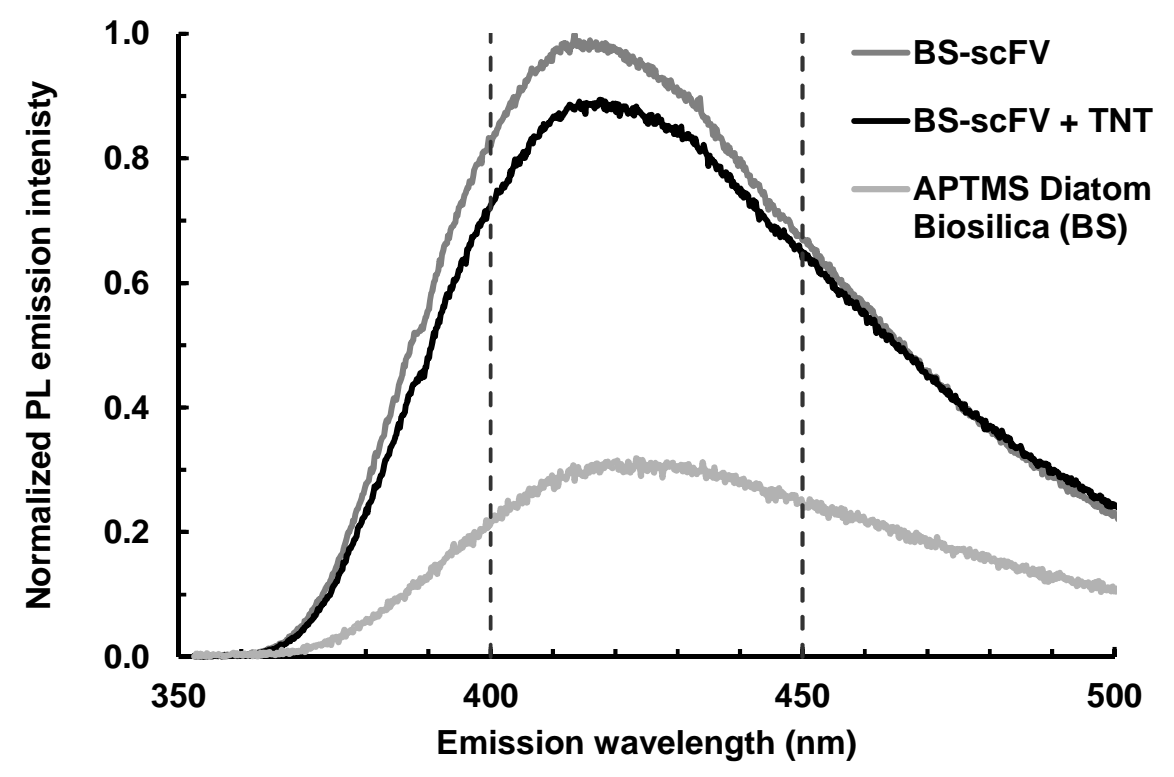


Fig. 4

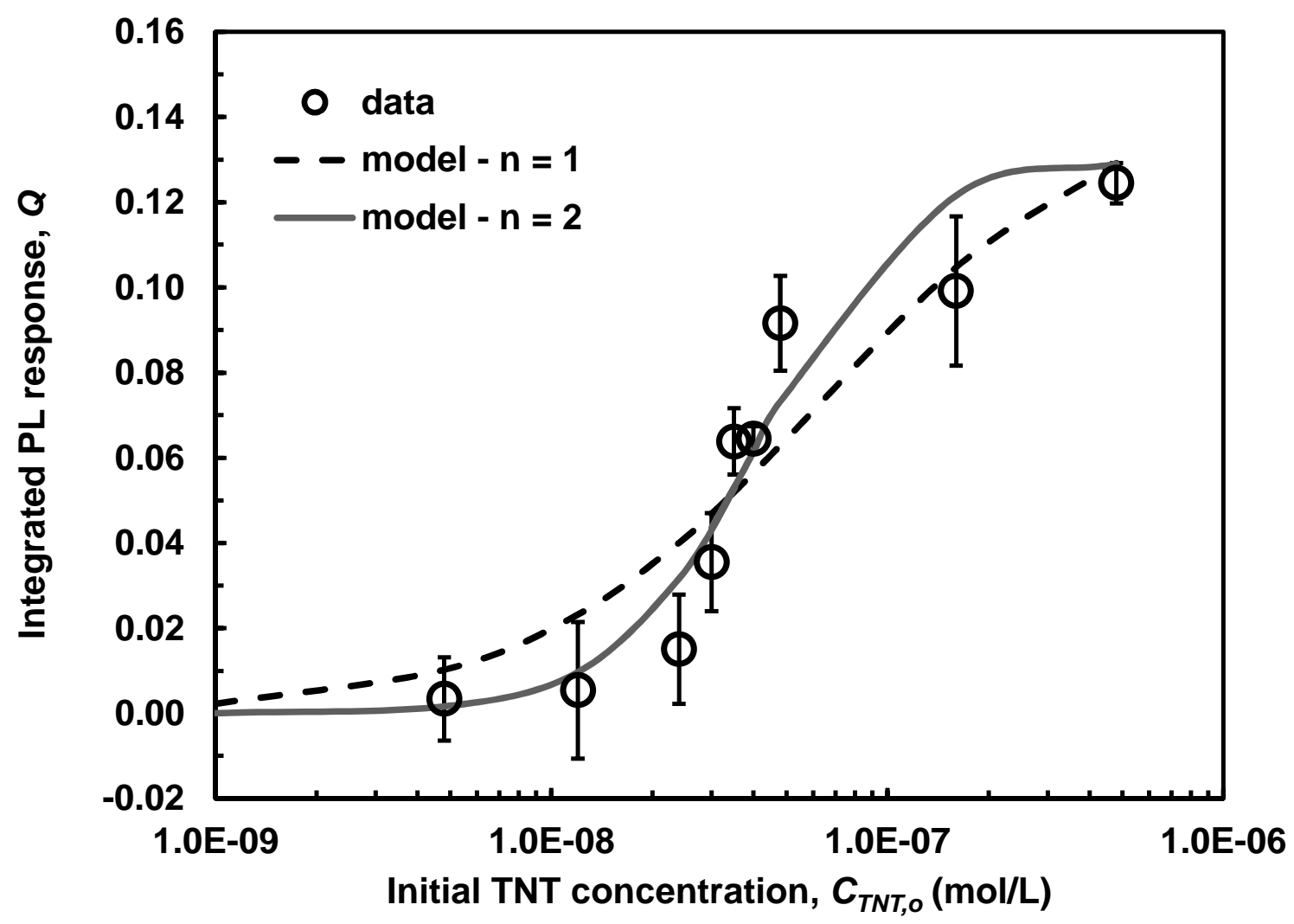


Fig. 5

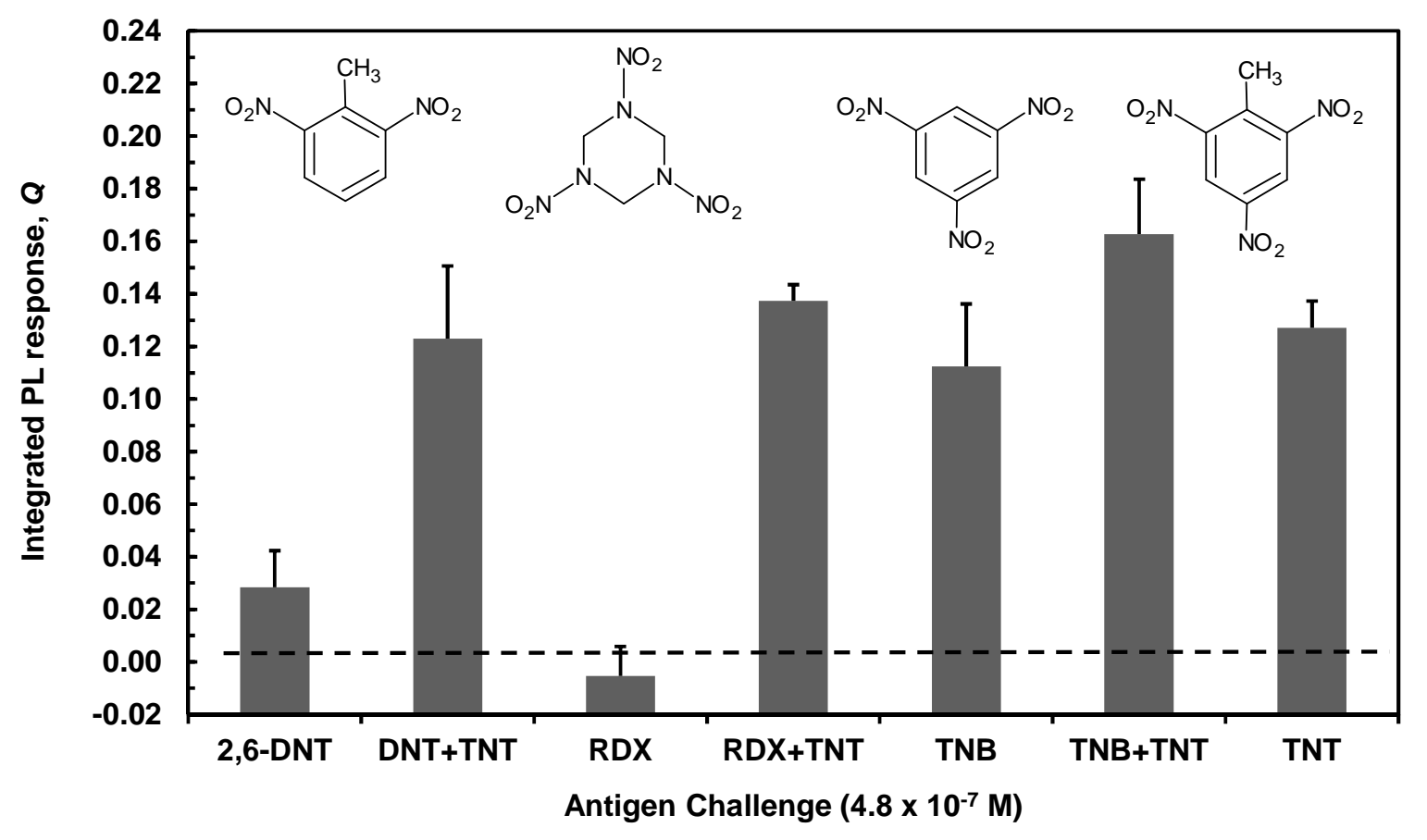


Fig. 6

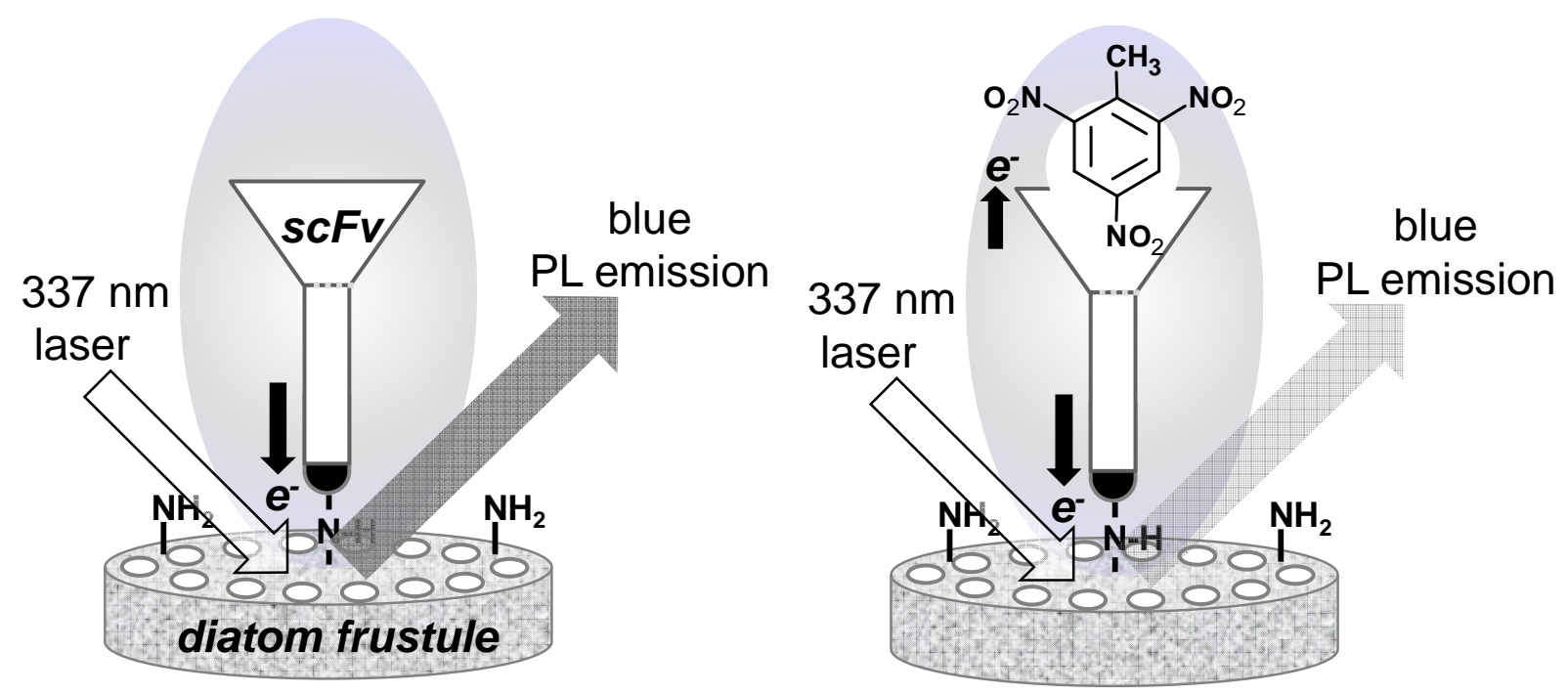

